\title{
From controlling to connecting: M'Wikwedong as a place of urban Indigenous health promotion in Canada
}

\author{
Carlos E Sanchez-Pimienta, Jeff Masuda, \& M'Wikwedong Indigenous
} Friendship Centre

\begin{abstract}
In recent years, health promotion has come under critique for being framed according to the contexts and priorities of Western communities, with the notion of 'control' underpinning much of its theoretical and practical development. Ceding space to Indigenous voices and knowledge is one way forward to overcoming this limitation and decolonizing the field. This paper reports on insights gained from a participatory digital storytelling project focused on Indigenous health promotion that took place at M'Wikwedong Indigenous Friendship Centre in the city of Owen Sound, Canada. The research team was formed by M'Wikwedong's Executive Director, five Indigenous youth, and two university researchers. We co-created data through an eight-month digital storytelling process that involved 13 weekly research meetings, the creation of four digital stories, and video screenings. We analyzed data from seven group interview transcriptions, field notes, and video transcripts through qualitative coding and theme building. The four themes we identified speak to the ways M'Wikwedong reinforced connections to youth, their sense of self, place in the city, and Indigenous cultures. From our findings, we theorize that egalitarianism of knowledge, restoring balance in relationships, and Indigenous leadership are core components of an 'ethos of connection' that underlies Indigenous health promotion. The 'ethos of connection' challenges Western notions of 'control' and brings attention to the unique expertise and practices of urban Indigenous communities and organizations as a primary basis for health promotion.
\end{abstract}

\section{The notion of 'control' in health promotion}

For the past half-century, health promotion has primarily been understood as "the process of enabling people to increase control over, and to improve, their health" (International Conference on Health Promotion, 1986). Initially, health promotion aimed to redirect attention away from clinical and curative health services toward broader social, environmental and political determinants of health, and more recently this discipline has been shifting research priorities, government policies, institutional practices, and public attitudes toward improving population health equity (Irvine et al., 2006). Throughout the evolution of health promotion as a distinctive field of theory and 
practice, the notion of 'control' comes to attention as a common thread. Indeed, the impetus on 'controlling health' can be traced back to one of the onto-epistemological assumptions of modern Western thought, the idea that "man was a rational being capable of mastering his environment, controlling his fate, and engineering his future" (Ahenakew et al., 2014).

Starting with health education, a significant antecedent of health promotion, pedagogic activities were mobilized to influence attitudes and behaviours with the hope of exerting control over the health of human groups (Green, 1999). Later, the nature of individual and small group interventions started to shift in the wake of the 'new social movements' in the 1960s and 1970s. During this period, oppressed communities around the world undertook collective action to demand political change rather than relying on political and professional stakeholders. Consequently, a tradition of controlling one's destiny emerged as a conceptual underpinning for community health promotion in the 1980s (Irvine et al., 2006). At this point, the concept of 'empowerment' led to a new set of interventions to control health, this time emphasizing community and collective political action (Labonte, 1994). With the formal consolidation of health promotion as an independent field of research and practice in the mid-1980s, the notion of control - both behavioural and sociopolitical - would become a defining underlying notion for this discipline for the next forty years.

Despite health promotion's seeming universal altruistic aims, the foundational economic (i.e. cost-saving) and moral (i.e. justice) arguments of this discipline have primarily been framed according to the needs and contexts of Western and industrialized countries (Nutbeam, 2008). Consequently, issues of colonial erasure within health promotion are gaining increased attention, such as the exclusion of Indigenous leadership and priorities in the Ottawa Charter and elsewhere in the field (McPhail-Bell et al., 2013; Corbin, 2016). Further, Indigenous communities across the world are resisting mainstream health promotion interventions that are perceived as externally imposed means to exert control over their lives (McPhail-Bell et al., 2015; Smylie, 2016). Fortunately, and with the aim of better recognizing the leadership and contributions of Indigenous communities, health promoters are coming together to show their public support. For instance, the International Union for Health Promotion and Education (IUHPE) released the Waiora Statement as a bold challenge to the health promotion community to take the voices and knowledge of Indigenous peoples seriously, for the benefit of all (IUHPE, 2019). There is thus an urgent need to lay the groundwork for fundamental transformations of the field, while also connecting its principles to the expertise grounded within specific Indigenous contexts. 


\section{The unique contributions and challenges of Indigenous health promotion}

Indigenous health promotion goes beyond making mainstream health promotion more 'culturally appropriate' and invites us to pay attention to the foundational ontoepistemological assumptions of this discipline (Brough et al., 2004). While diverse in historical context and cultural orientation, forms of Indigenous health promotion around the world stem from ancestral knowledge and spiritual practices embedded in Indigenous ways of knowing and living (Ratima et al., 2019). Indigenous knowledge systems are often underpinned by relational onto-epistemologies that see reality as an intricate process of relationships between people, the land, objects, ideas, and metaphysical entities (Wilson, 2008; Ahenakew, 2014); and human health as profoundly interconnected with the health of the land (Durie, 20014; IUHPE, 2019). Whereas the onto-epistemological assumptions of mainstream health promotion emphasize excreting control over health, Indigenous health promotion initiatives place greater emphasis on relationality and supporting values such as respect, self-determination, and nonjudgemental approaches to service delivery (Van Herk et al., 2012; Yi et al., 2015). For example, previous collaborative work with Indigenous youth shows that successful Indigenous health promotion initiatives have focused on fostering relationships with the community, promoting land-based activities, and supporting cultural revitalization (Riecken et al., 2006; Big-Canoe and Richmond, 2014; Hatala et al., 2019).

While there are many examples of Indigenous health promotion initiatives across the world (Smylie et al., 2016; MacLean et al., 2017; Murdoch-Flowers et al., 2019), advancing this agenda has been difficult within urban contexts, particularly given the global trend toward Indigenous urbanization. In countries like Canada, many cities continue to uphold segregated geographies of entrenched racism, whether in the context of interpersonal interactions, systemic racism, or the dominance of built urban infrastructure that does not account for Indigenous priorities or worldviews (Peters, 2002). Thus, the present pattern of colonial urbanization makes the city a challenging setting for urban Indigenous communities to connect with, affirm, and re-create forms of Indigenous health promotion. In this paper, we examine one case of urban Indigenous leadership at the M'Wikwedong Indigenous Friendship Centre ('M'Wikwedong' from here on). We focus on understanding how an Indigenous Friendship Centre can facilitate health promotion from the perspective of Indigenous community members who experience its supports. This manuscript originates from the first author's master's thesis (Sanchez-Pimienta, 2018) and responds to the Waiora Statement (2019) by further elaborating on the implications of mobilizing an ethos of connection, rather than control, in health promotion. Our paper seeks to contribute to stimulating health promotion's conceptual and practical development going forward in ways that are 
appropriate to the current socio-political climate, nearly 35 years after the Ottawa Charter.

\section{Methodology}

\section{Research setting}

Our research took place in Owen Sound, Ontario, on the traditional territory of Saugeen First Nation and Chippewas of Nawash Unceded First Nation, which are collectively known as Saugeen Ojibway Nation (SON). Owen Sound was built in an area that once hosted the largest settlement of Indigenous peoples in the local region but was displaced through Treaty 82 in 1857, arguably signed under unfair circumstances (McMullen, 1997). As is the case across Canada, Indigenous communities have eventually found their way back to their home territories; among the 21,341 inhabitants of Owen Sound, 890 reported Aboriginal identity in 2016 (Statistics Canada, 2019). Owen Sound is the largest city and the regional hub for education, employment, and social services within a mostly rural region comprised of the Grey and Bruce Counties, and the SON reserves, making M'Wikwedong an accessible resource for the region's Indigenous inhabitants.

M'Wikwedong started operations in 1988 and was incorporated as a Friendship Centre in 2001. Although the 'Friendship Centre Movement' has a long legacy of grassroots innovation in health and wellbeing, these organizations have rarely garnered the attention of health promotion scholars and practitioners, with some exceptions (Lavallée, 2008; Hayhurst et al., 2015). At the time of fieldwork in 2018, four urban Indigenous organizations operated in Owen Sound, among which M'Wikwedong was the oldest. This Friendship Centre's mandate includes serving the Indigenous inhabitants of the Grey Bruce region - particularly those who experience poverty or other forms of socioeconomic marginalization - and breaking down systemic barriers between local Indigenous and non-Indigenous peoples. The majority of M'Wikwedong's staff were Indigenous. They delivered nine programs that focused on community wellness, healthy child development, family supports, youth supports, and cultural programming, with some activities open to non-Indigenous participants. Despite M'Wikwedong's intensive and longstanding work, professionals in the social service and health sectors in Grey Bruce had limited awareness of the programming and impact of this Friendship Centre, in part due to inter-organizational barriers for collaboration (Sanchez-Pimienta, 2019).

\section{Research initiation}

The research project reported herein is part of an ongoing collaborative relationship between M'Wikwedong and the Centre for Environmental Health Equity 
(CEHE), a community-based participatory research program led by the second author, Jeff Masuda, and based at Queen's University in Kingston, Canada. In 2016, existing CEHE partners in Owen Sound suggested that M'Wikwedong was a relevant organization to engage with due to its longstanding work with the urban Indigenous community. The first author, Carlos Sanchez-Pimienta, visited M'Wikwedong in January 2017 to introduce himself and explore the opportunity of conducting a participatory action research project that would directly support their current programming priorities. Carlos followed-up through phone calls with M'Wikwedong's Executive Director to further discuss his relevant professional experience and the potential research designs. M'Wikwedong kindly granted CEHE the opportunity to work with them as host and advisor on this research project. The first author resided in Owen Sound for the entire 16-month duration of the project, working to co-design and facilitate the research with the local team. Jeff visited regularly and received frequent updates on the team's progress. Adhering to principles of reciprocity in participatory and Indigenous research, Carlos volunteered as a gesture of gratitude to M'Wikwedong for approximately 30 hours per week during summer 2017, and 2-8 hours weekly during the researchintensive phase of the project.

Through volunteer work and participation in M'Wikwedong's programming, Carlos identified a group of youth that was well-positioned to become co-researchers on this project, as they were consistent program participants and actively supported community-wide programming through volunteer work at the Centre. The first author shared his desire to co-design a research project that would directly support their interests and aspirations and explained the related participation tasks and time commitment. Three youth confirmed their willingness to take part in creating such a project. In July and August 2017, Carlos facilitated a series of informal conversations with these youth to brainstorm ideas for research design. The facilitation of informal discussions took on a strength-based approach to identify research methods that built on youth's skills, creativity and leadership; and research topics that brought more attention to community assets and were relevant to health promotion research. These conversations took place at M'Wikwedong and invited Elders, staff, and other community members to provide their input.

Research design

Indigenous approaches to research (Wilson, 2008; Ontario Federation of Indigenous Friendship Centres, 2016) provided ethical and relational guidance, and Participatory Action Research (Kindon et al., 2007) offered procedural direction to plan emergent research processes such as that the research process would have transformative potential. In August 2017, the youth co-researchers expressed an 
interest in creating a project that would focus on M'Wikwedong's importance in their lives. They elected to employ video-making techniques as they hoped to create promotional videos that would spread awareness about M'Wikwedong's value to the broader community. Our research project adopted an exploratory case study design (Yin, 2003) guided by the research question: How does M'Wikwedong foster health in the city of Owen Sound from the perspective of young Indigenous peoples? A case study design provided flexibility for the youth co-researchers to conduct an iterative and in-depth exploration of the health-promoting processes that were most important from their perspective. For this case study, 'health' and 'health promotion' were open constructs for youth co-researchers to discuss and work with on their terms.

\section{Research team}

The research team is represented in the authorship of this paper. 'M'Wikwedong Indigenous Friendship Centre' stands for the five young Indigenous researchers, and the Centre's Executive Director, Renee K. Abram. We set a limit of up to five youth-co researchers to privilege in-depth data co-creation. Among youth co-researchers, James Schlonies, Ryerson King and Steven Schlonies, joined at the stage of project design. Two other youths, Ciph Harrisson and Nikita Jones, joined the research team through snowball recruitment in January 2018. Co-researchers were 17-22 years old as of May 2018. They had diverse ancestral backgrounds, including Ojibway, Cherokee, Cree and Métis. Some worked or studied, others did both, and one performed care work for his family. Their participation in M'Wikwedong's programming ranged between two and ten years, which positioned them as experts about M'Wikwedong's work. For the youth involved, being a co-researcher implied taking part in all stages of the research process they chose to, including design, implementation, analysis, and knowledge translation. M'Wikwedong sponsored co-researchers with honoraria for their work on this project ( $\$ 15$ CAD per hour). From CEHE's side, Jeff is an established researcher involved in Canadian and international health promotion contexts. Jeff provided ongoing feedback at all stages of the research process, advised on the theoretical positioning of research findings, and provided oversight for methodological robustness and rigour. Carlos, an international student at the time, facilitated this research as part of his master's thesis. Carlos identifies as a Mexican mestizo with both Indigenous and Spanish ancestry but was raised as a non-Indigenous person in a Mexican context that is characterized by internal colonialism. In recognition of his positionality as a non-invited settler in Canada, Carlos strove to maintain a position of humility in facilitating this research journey under the guidance of M'Wikwedong through eight months of on-site research preparation in 2017. 


\section{Data gathering and analysis}

This project employed digital storytelling as a method for knowledge co-creation and documentation. Digital storytelling is a facilitated technique that guides participants in identifying an idea, transforming it into a compelling story, creating a video script and storyboard, gathering audiovisual media, and editing a video that is typically 3-5 minutes long (Lambert, 2010). Digital storytelling gives video-makers control over the topic that is being researched, with each video-maker creating their research-story. The digital storytelling process took place through 13 weekly research meetings held at M'Wikwedong over five months, plus three months of knowledge translation activities in 2018. During each research team meeting, we worked on the research from 20 to 60 minutes, followed by 20-90 minutes of unstructured 'fun' time, as suggested by coresearchers to foster a balance between research work and social time.

We used research team meetings to identify positive experiences that youth coresearchers attributed to their affiliation with M'Wikwedong. Our team discussed how these experiences intersected with their understandings of health and wellbeing and their views on M'Wikwedong's health promotion work. The team's insights were documented through seven semi-structured group interviews that took place at seven of our 13 research team meetings. Interviews were audio-recorded and transcribed verbatim. The first author used note-taking to record follow-up questions about the digital storytelling projects and the team's ideas for improving the research process facilitation. [name1] drew from his observations, intuition and co-researchers' suggestions to identify pertinent information for research notes. In addition to research team meetings, each youth co-researcher required personalized mentorship on scriptwriting, storyboard design, media gathering, and video editing. To meet these needs, our team implemented a total of 22 one-on-one sessions, where each youth coresearcher engaged in five to seven sessions ranging from 30 minutes to three hours. The creative process was completed when all youth co-researchers finished the editing of their videos and identified relevant community events for their screening.

For data analysis, we drew on Charmaz's (2008) approach by iteratively coding data and building themes that spoke M'Wikwedong's health promotion processes. Carlos led the coding and theme building process using NVivo (version 12, QSR International). The initial round of coding involved a line-by-line reading of interview transcripts, video scripts and research notes, to create descriptive codes that our team used to further sort and synthesize the data. The second round of coding involved comparing the initial codes to identify overarching themes across the three groups of research data. Carlos used research team meetings to discuss and refine preliminary themes with the research team. Youth co-researchers assented to the identified 
themes, updated their names and pronouns, and suggested terms that better represented their lived experiences. Our team concluded the data gathering process when youth co-researchers posited that they had nothing else to add to our discussion about the health-promoting value of their videos. Similarly, data analysis concluded when youth co-researchers agreed that our identified themes accurately represented their experiences and views on M'Wikwedong's work. At this point, our analysis identified four themes that centred on safety, making space for youth in the city, increasing access to traditional Indigenous teachings, and supporting youth's leadership. This paper presents a further iteration of theme building that reorganized previously identified codes and themes to better reflect the type of connections that M'Wikwedong fostered, considering our discussion of health promotion as controlling or connecting.

\section{Ethical considerations}

Our research process adhered to the Utility, Self-Sufficiency, Access and Interrelationality (USAI) research framework for conducting research with urban Indigenous communities (Ontario Federation of Indigenous Friendship Centres, 2016). Utility requires that research results directly benefit the Indigenous community driving the process. In this case, youth co-researchers strengthened their video-making and research skills and created videos that they and M'Wikwedong could use for public relations. Self-voicing recognizes that Indigenous knowledge is authored and owned by Indigenous communities. Digital stories and related publications were authored by their youth creators or M'Wikwedong; videos are owned by their youth creators. Access speaks to the importance of having readily understandable research outputs for community members. This principle was achieved by sharing research findings through youth's videos. Finally, Inter-relationality positions research as part of a historical and geopolitical context. Guided by this principle, our overall research design aimed to intervene in a context where Indigenous health promotion has been frequently overlooked within established public health institutions and practices. Additional ethical considerations for this project were compiled in a research agreement between M'Wikwedong and CEHE that provided a detailed account of ownership, control, access, possession and safe storage of research data. Ongoing consent from youth coresearchers was pursued through a combined Letter of Information and Consent Form. All youth co-researchers agreed to use their names and waived confidentiality. The research obtained clearance from the General Research Ethics Board of Queen's University in December 2017. 


\section{Findings}

The project produced four videos, ranging from one to four minutes in duration. One co-researcher chose not to undertake a video, two co-researchers created private videos, and two co-researchers created public videos (available at https://youtu.be/9SiLCMiZ2zQ and https://youtu.be/kQoCAln9y10). Drawing from our previous critique of Western health promotion's ethos of epistemic control set against an Indigenous ethos of health promotion as relational connection, this section presents the findings of our research team through four inter-related themes that speak to the relational processes that youth described in their research-story journeys, focusing on their connections to place and community. The four themes illustrate how M'Wikwedong emphasized relationality in cultivating relationships with youth, supporting youth to connect with their sense of self, providing access to Indigenous programming, and fostering more opportunities for youth to feel valued within Owen Sound.

\section{Connecting youth with M'Wikwedong}

Youth co-researchers shared stories of how they established a long-standing relationship with M'Wikwedong and contextualized those experiences with previous unsuccessful attempts of other organizations. Ciph's video is about their journey discovering themself as a two-spirit person and looking for social urban environments where a feeling of belonging was nurtured. After living in two different cities, this coresearcher noticed that it was difficult to find service providers that felt safe for two-spirit youth. The climax moment of their video arrives when Ciph 'comes-out' as two-spirit to both M'Wikwedong's Men's and Women's Drum Circles. This co-researcher felt embraced by M'Wikwedong's leadership staff and the wider community. In fact, both drum groups performed songs for Ciph to include in their video, augmenting their hope of living in a community where they could fully participate. The experience of safely 'coming-out' within the context of M'Wikwedong and its membership was a life-marking event that was defining for Ciph's sense of wellness in the community and the broader city.

Co-researchers stressed that voluntarism was a second essential aspect for sustaining trusting relationships between social service organizations and Indigenous youth. Co-researchers drew from their lived experience to contrast M'Wikwedong's approach from the more coercive environments of non-Indigenous social and health services. For instance, James recounted how in their childhood, they realized some programs were voluntary for parents but not for their children. Co-researchers affirmed that when a change was forced upon youth, it was perceived as a threat. Coresearchers preferred to be asked whether they wanted help, and what it should look like. Nikita exemplified with her experience with the Wasa-Nabin youth program: 
"I have a lot of discussions with him [Wasa-Nabin Program Coordinator] because that's what I wanted to. He asked me what I wanted from him. And I said discussions, ask me how l'm doing (...) and just break it down." (Nikita, interview 1).

Ensuring voluntary and non-judgemental support allowed M'Wikwedong to be successful in sustaining relationships with youth co-researchers. By connecting with M'Wikwedong, youth were able to access diverse experiences that did not attempt to create pre-determined health outcomes but rather to support them in their journeys, needs and desires.

\section{Connecting youth with their sense of self}

The trusting relationships between M'Wikwedong and youth co-researchers created synergic opportunities to work on developing increased connections with their sense of self. For instance, James emphasized how M'Wikwedong had helped them to find their voice and share it with others. James' video conveys their journey coping with mental health difficulties in their childhood. After seeking supports at diverse social service organizations, this co-researcher discovered M'Wikwedong, describing the difference as follows:

"At the Centre [M'Wikwedong], drumming helped me to express myself. Whereas before, I didn't defend myself, and I didn't do anything about it. I was not speaking up for myself. It's hard to explain how it works [drumming] if you haven't experienced it for yourself. But it is very powerful to hear someone talking about it, and sharing teachings, and then to do it yourself." (James, video)

At the end of their video, James states that they are working to support other Indigenous youth in their community by sharing their experience and advocating for other youth at M'Wikwedong's Board of Directors. James' story illustrates the ability of M'Wikwedong to support youth in connecting with their passions, facilitating a sense of purpose, and offering opportunities to pursue their dreams.

Youth co-researchers also highlighted how M'Wikwedong provided them with opportunities to see themselves as leaders. At the time of data co-creation, coresearchers were working on youth-led activities that included promoting the formation of a youth council. For instance, youth co-researchers organized events, such as a pizza social and a community dance to reach out to other Indigenous youth. Further, Ciph and James advocated with staff and community members to bring awareness about the importance of having supports for two-spirit youth. As a result, M'Wikwedong staff members worked with them toward bringing traditional knowledge holders who could share teachings about two-spirit people with the community. By giving space for youth to connect with their ideas and leadership capacity, M'Wikwedong was able to support co-researchers' desires and priorities for creating healthier urban environments. 


\section{Connecting youth with Indigenous cultures}

Youth co-researchers stressed how M'Wikwedong performed a vital contribution to their health and wellbeing by connecting them with Indigenous programming focused on their cultures. As a youth living off-reserve, James affirmed they had few opportunities to experience their own culture. They claimed that at school and most places in Owen Sound, they were socialized through Euro-Canadian values and worldviews. At M'Wikwedong, youth had access to a wide variety of Indigenous cultural programming, including traditional tool-making, drumming, and beading. Youth also learned about the traditional Indigenous teachings associated with each activity. For example, Ryerson gathers cedar in his video and then puts down a tobacco offering to thank the land for what he took, as an example of honouring the reciprocal relationships that exist between the land and people. Ryerson reflects on his video about the teachings he learned at M'Wikwedong and the meaning such teachings have brought to his life:

"I think learning about the Ojibway culture taught me more about my background, how we did things back then, and how to respect others. Our culture gives us a different point of view. It's like more understanding of what we're getting, and what we're receiving, and what we're doing." (Ryerson, video)

Indigenous programming provided other youth co-researchers with similar opportunities. Ceremonies at M'Wikwedong allowed co-researchers to learn more about traditional roles and responsibilities they could engage in as part of the urban Indigenous community. Youth co-researchers also experienced forms of healing that restored spiritual and emotional balance in their lives. For example, Ciph highlighted the spiritual contributions of the song they chose for their video, which called upon the bear spirit to ask for the strength and courage they needed to share their story as a twospirited person. It was clear that such cultural programming was a defining reason for choosing where to live: "Without M'Wikwedong, there wouldn't be much of any opportunity for me [in Owen Sound]" (Ciph, interview 6).

Connecting youth with the city

M'Wikwedong provided youth with opportunities to take part in events and activities at locations across Owen Sound that they would usually not visit. Nikita's digital storytelling video was evident in this regard. At the time of this research, Nikita had recently moved to Owen Sound to work while she applied for college. She became involved with M'Wikwedong because of a youth program it delivered at her high school. Nikita's video shares a story about how M'Wikwedong supported her in having a safe place to go within the city. In discussing her video, Nikita affirmed that the Friendship Centre provided a hub to connect with other Indigenous youth and community 
members. As the research process unfolded, Nikita was able to develop strong friendships with other Indigenous youth she met at M'Wikwedong.

For the other co-researchers, M'Wikwedong provided additional supports to take part in community events happening around the city where they had opportunities for meaningful participation, such as drumming for event openings and closings. Between 2017 and 2018, co-researchers took part in numerous events hosted at community centres, churches, parks, libraries, colleges, resorts, music halls, and reserves. The visible leadership roles that youth co-researchers had at open community events filled youth with pride and affirmed the value of their Indigeneity as an integral part of the city's cultural fabric.

\section{Knowledge translation}

Informed by the notion of Aboriginal knowledge translation (Estey et al., 2009), our project allowed for multidirectional knowledge sharing processes among the research team, M'Wikwedong staff, and the wider public. Nikita and Ryerson shared their videos only with the community within M'Wikwedong by screening them at the Centre's 2018 Annual General Meeting. At this event, community members expressed their pride in the work of youth co-researcher and staff affirmed that these videos helped them to better understand the importance of their daily work for Indigenous youth. James and Ciph made their videos publicly available and personally presented their videos in at least five events, which included film festivals, a professional conference, and an Indigenous-led event. One highlight is that Ciph's and James' videos were given awards in the 'senior' and 'LGBTQ+' categories at the 2018 Grey Bruce Youth Film Festival. Additionally, their videos were posted on M'Wikwedong's website and published in an Indigenous journal (Harrisson et al., 2019; Schlonies et al., 2019).

\section{Discussion}

Our digital storytelling approach allowed for a prolonged, iterative and reflective exploration of how M'Wikwedong's work in the city of Owen Sound represents an exemplar of Indigenous health promotion from the perspective of Indigenous youth. We have identified four core themes that emphasized building and sustaining connections with M'Wikwedong, youth development opportunities, culturally-specific programming, and community-wide events. Facing a colonial urban context and a regional public health mentality largely premised on Western values and priorities, M'Wikwedong focuses on re-establishing and nourishing relationships that have been damaged by colonization processes that remain a fixture of the city's social and physical landscape. The four inter-related themes that we presented illuminate the sets of relationships that were most salient for urban health and wellbeing from the perspective of youth co- 
researchers. The inter-relation among the four themes was evident in the lived experience of youth co-researchers, including their participation in the research process. For example, Ciph's video illustrates two forms of connection by showing how M'Wikwedong nurtured a trusting relationship with them (i.e. connection with M'Wikwedong) and emphasizing that the availability of Indigenous cultural programming was a determining factor for Ciph's sense of wellness in Owen Sound (i.e. connection with culture). Beyond the content of their video, the digital storytelling process became an additional opportunity for Ciph to affirm their sense of self as a two-spirit person (i.e. connection with the sense of self) and to assert the value of M'Wikwedong's health promotion leadership within the Grey Bruce area through video presentations (i.e. connection with the city).

Our work builds on previous research in health promotion in collaboration with Indigenous youth, which has also contributed to re-imagining new possibilities for health promotion (Riecken et al., 2006; Flicker et al., 2014). Taken in isolation, the four forms of connection that youth co-researchers prioritized affirm previous Indigenous health promotion perspectives. For instance, Smylie et al. (2016) showed that forging trusting relationships and making space for Indigenous self-determination are markers of successful Indigenous health promotion programming; Yi et al. (2015) and Hatala et al. (2017) highlighted the need for health promotion programming that empowers youth to affirm their self-confidence and unique skills; Durie (2004), Riecken et al. (2006), and Big-Canoe and Richmond (2014) emphasized the importance of traditional Indigenous knowledge and spiritual practices for Indigenous peoples' sense of health and wellness; and Skinner and Masuda (2013) and Hatala et al. (2019) pointed to the unique challenges and opportunities for urban Indigenous youth to connect with nature and other environmental assets within the city. The unique contribution of our findings is better appreciated, however, when considering the relatedness of the four types of connection identified by youth co-researchers, and their relationship with our presentation of the onto-epistemological assumptions that underlie Indigenous and mainstream health promotion.

Our research confirms how M'Wikwedong's approach to health promotion embodies an ethos of connection, which is significantly different from the ethos of control that has characterized Western health promotion. We suggest three critical components of this ethos of connection. First, an ethos of connection aligns with principles of egalitarianism and inclusiveness of knowledge (Wilson, 2008). As found in previous publications (Yi et al., 2015; Hatala et al., 2017), youth co-researchers in this project reclaimed equal opportunities to define what could work, or not, as healthpromoting for the context of their lives vis à vis the professional knowledge that 
underpinned mainstream health promotion programming. This aspect of the ethos of connection points to the need to make significant space for open-ended health promotion approaches that address community and youth priorities as they are, rather than force-fitting local programs into externally-determined health outcomes. We suggest that a 'letting go' of pre-determined topic foci of conventional health promotion approaches (i.e. chronic disease prevention) does not mean that such priorities are sidelined. Instead, we may find an improved efficacy of such 'secondary' outcomes to the primary (and collective) health outcomes of strengthened dignity, revitalized language and culture, asserted self-determination, and a closer connection to land and community.

Second, an ethos of connection focuses on restoring the balance in a set of interconnected relationships that are meaningful for youth, such as connections to the land, community, culture, language, and spirituality (Riecken et al., 2006; Big-Canoe and Richmond, 2014; Hayhurst et al., 2015; Hatala et al., 2017; MacLean et al., 2017). This paper adds to this literature, showing that M'Wikwedong improved youth's access to events and places in the city that are otherwise difficult to access in a context of social isolation, poverty, and anti-Indigenous racism. Youth co-researchers were then more able to establish social relationships within the community and foster increased awareness about the ongoing contributions of Indigenous peoples and organizations in the region. These findings suggest that health promotion programming ought to be oriented not only toward ostensible 'beneficiaries,' but rather explore more reciprocal relationships between the recipients of program delivery, and their contributions to the broader community.

Third, an ethos of connection is supported by strong Indigenous leadership. In the context of this case study, youth co-researchers' stories were enabled by M'Wikwedong - an exemplar of the Friendship Centre movement in Canada. Although youth were able to combat deficit-based representations of Indigenous peoples and their institutions in direct engagements with the general public and health professionals, youth co-researchers would likely have encountered significant challenges in doing so in the absence of organizations like M'Wikwedong. Our research findings resonate with previous calls to support the organizational capacity and leadership of urban Indigenous organizations (Brough et al., 2004) to better equip sustained work towards dismantling the structural forces that perpetuate anti-Indigenous racism and resulting health inequities.

Importantly, the notion of 'control' is not absent in Indigenous health promotion. Indigenous control over funding, planning, deriving and evaluating health promotion initiatives appears in the published literature as an explicit way to interrupt further 
colonial interventions that undermine Indigenous self-determination (McPhail-Bell et al., 2015; Smylie et al., 2016). However, we posit that such for of control attempts to resist, rather than advance, efforts to engineer pre-determined health and wellness outcomes, as characteristic of the 'ethos of control' of mainstream health promotion. Overall, the insights of our research team about M'Wikwedong's health promotion work do not intend to define what an ethos of connection looks like for all forms of Indigenous health promotion. Rather, we provided an initial theorization of what an 'ethos of connection' can mean based on the context of urban Indigenous organizations like M'Wikwedong.

We invite others to embark upon or to continue the journey of humble knowledge sharing, where Western health promotion stakeholders take the space, time, and means to listen to the profound contributions of urban Indigenous communities and organizations as a basis for health promotion. In exploring the unique features of Indigenous health promotion, Indigenous scholars and community members are best positioned to examine these processes in terms of their Indigenous knowledge systems. This is a task that could either be done through nation-specific onto-epistemologies or by exploring commonalities across diverse Indigenous knowledge-systems. NonIndigenous scholars can strengthen their capacity to demonstrate allyship with Indigenous communities, organizations or academics, further investigate the limitations of the onto-epistemological assumptions of Western health promotion approaches and make more space for prioritizing the contributions of Indigenous health promotion in educational, professional and practice settings.

\section{References}

Ahenakew, C., Andreotti, V. D. O., Cooper, G. and Hireme, H. (2014) Beyond epistemic provincialism: De-provincializing Indigenous resistance. AlterNative: An International Journal of Indigenous Peoples, 10, 216-231.

Big-Canoe, K. and Richmond, C. A. M. (2014) Anishinabe youth perceptions about community health: Toward environmental repossession. Health and Place, 26, 127-135.

Brough, M., Bond, C. and Hunt, J. (2004) Strong in the city: Towards a strengthbased approach in Indigenous health promotion. Health Promotion Journal of Australia, 15, 215-220.

Charmaz, K. (2008) Grounded theory as an emergent method. In Hesse-Biber, S. N.

Leavy, P. (eds), Handbook of Emergent Methods, 2nd edition, Chapter 4. Guildford Press, New York, pp. 155-170. 
Corbin, J. H. (2016) Health promotion research: Thinking critically about knowledge production. Health Promotion International, 31, 739-741.

Durie, M. (2004). An Indigenous model of health promotion. Health Promotion Journal of Australia. 15, 181-185.

Estey, E., Smylie, J. and Macaulay, A. (2009) Aboriginal knowledge translation: Understanding and respecting the distinct needs of Aboriginal communities in research. Ottawa. http://www.cihr-irsc.gc.ca/e/8668.

Flicker, S., Danforth, J., Wilson, C., Oliver, V., Larkin, J., Restoule, J. et al. (2014). "Because we have really unique art": Decolonizing research with Indigenous youth using the arts. International Journal of Indigenous Health, 1, 16-34.

Green, L. W. (1999) Health education's contributions to public health in the twentieth century: A glimpse through health promotion's rear-view mirror. Annual Review of Public Health, 20, 67-88.

Harrisson, K. L., M'Wikwedong Youth Group, Sanchez-Pimienta, C. E. and Masuda, J. (2019) Thunder Finder. First Peoples Child \& Family Review, 13, 9.

Hatala, A. R., Pearl, T., Bird-Naytowhow, K., Judge, A., Sjoblom, E. and Liebenberg, L. (2017). "I have strong hopes for the future": Time orientations and resilience among Canadian Indigenous youth. Qualitative Health Research, 27, 1330 1344.

Hatala, A. R., Morton, D., Njeze, C., Bird-Naytowhow, K. and Pearl, T. (2019). Re-imagining miyo-wicehtowin: Human-nature relations, land-making, and wellness among Indigenous youth in a Canadian urban context. Social Science and Medicine, 230, 122-130.

Hayhurst, L. M. C., Giles, A. R. and Radforth, W. M. (2015) 'I want to come here to prove them wrong': Using a post-colonial feminist participatory action research (PFPAR) approach to studying sport, gender and development programmes for urban Indigenous young women. Sport in Society, 18, 952-967.

International Conference on Health Promotion (1986) Ottawa Charter for Health Promotion. Ottawa: World Health Organization, Health and Welfare Canada, Canadian Public Health Association.

Irvine, L., Elliott, L., Wallace, H. and Crombie, I. K. (2006) A review of major influences on current public health policy in developed countries in the second half of the 20th century. Journal of The Royal Society for the Promotion of Health, 126, 73-78.

IUHPE (2019) Waiora - Indigenous Peoples' Statement for Planetary Health and Sustainable Development. Rotorua: IUHPE World Conference on Health Promotion. 
Kindon, S., Pain, R. and Kesby, M. (2007) Participatory action research approaches and methods. Connecting people, participation and place. Oxon: Routledge.

Labonte, R. (1994) Health promotion and empowerment: Reflections on professional practice. Health Education Quarterly, 21, 253-268.

Lambert, J. (2010) Digital storytelling cookbook. Berkeley: Digital Diner Press.

Lavallée, L. (2008) Balancing the medicine wheel through physical activity. Journal of Aboriginal Health, 4, 64-71.

MacLean, S., Ritte, R., Thorpe, A., Ewen, S. and Arabena, K. (2017) Health and wellbeing outcomes of programs for Indigenous Australians that include strategies to enable the expression of cultural identities: A systematic review. Australian Journal of Primary Health, 23, 309-318.

McMullen, S. (1997) Disunity and dispossession: Nawash Ojibwa and Potawatomi in the Saugeen territory, 1836-1865. University of Calgary.

McPhail-Bell, K., Fredericks, B. and Brough, M. (2013) Beyond the accolades: A postcolonial critique of the foundations of the Ottawa Charter. Global Health Promotion, 20, 22-29.

McPhail-Bell, K., Bond, C., Brough, M. and Fredericks, B. (2015) 'We don't tell people what to do': Ethical practice and Indigenous health promotion. Health Promotion Journal of Australia, 26, 195-199.

Murdoch-Flowers, J., Tremblay, M. C., Hovey, R., Delormier, T., Gray-Donald, K., Delaronde, E., et al. (2019) Understanding how Indigenous culturally-based interventions can improve participants' health in Canada. Health Promotion International, 34, 154-165.

National Association of Friendship Centres (no date) History. http://nafc.ca/en/who-we-are/history/ (last accessed 29 June 2018).

Nutbeam, D. (2008) What would the Ottawa Charter look like if it were written today? Critical Public Health, 18, 435-441.

Ontario Federation of Indigenous Friendship Centres. (2016) USAI research framework. Toronto.

Peters, E. J. (2002) 'Our city Indians': Negotiating the meaning of First Nations urbanization in Canada, 1945-1975. Historical Geography, 29, 75-92.

Ratima, M., Martin, D., Castleden, H. and Delormier, T. (2019) Indigenous voices and knowledge systems - promoting planetary health, health equity, and sustainable development now and for future generations. Global Health Promotion, 26, 3-5. 
Riecken, T., Scott, T. and Tanaka, M. T. (2006) Community and culture as foundations for resilience: Participatory health research with First Nations student filmmakers. Journal of Aboriginal Health, 3, 7-14.

Sanchez-Pimienta, C. E. (2018) Promoting healthy urban environments for young Indigenous peoples: The case of M'Wikwedong Native Cultural Resource Centre. Queen's University.

Sanchez-Pimienta, C. E., and Masuda, J. (2019) The Giiwe Project. Evaluation Report. Kingston, Canada: Centre for Environmental Health Equity.

Schlonies, J., M'Wikwedong Youth Group, Sanchez-Pimienta, C. E. and Masuda, J. (2019) Animkee. First Peoples Child \& Family Review, 13, 10.

Smylie, J., Kirst, M., McShane, K., Firestone, M., Wolfe, S. and O'Campo, P. (2016) Understanding the role of Indigenous community participation in Indigenous prenatal and infant-toddler health promotion programs in Canada: A realist review. Social Science and Medicine, 150, 128-143.

Statistics Canada (2019) Census Profile, 2016 Census - Canada [Country] and Owen Sound, City [Census subdivision], Ontario. http://www12.statcan.gc.ca/censusrecensement/2016/dp$\mathrm{pd} /$ prof/details/page.$c f m$ ?Lang=E\&Geo1=PR\&Code1=01\&Geo2=CSD\&Code2=354205 9\&Data=Count\&SearchText=Owen Sound\&SearchType=Begins\&SearchPR=01\&B1=All (last accessed 12 September 2017).

Van Herk, K. A., Smith, D. and Tedford Gold, S. (2012). Safe care spaces and places: Exploring urban Aboriginal families' access to preventive care. Health and Place, 18, 649-656.

Wilson, S. (2008) Research is ceremony: Indigenous research methods. Black Point: Fernwood Publishing.

Yi, K. J., Landais, E., Kolahdooz, F. and Sharma, S. (2015) Factors influencing the health and wellness of urban aboriginal youths in Canada: Insights of in-service professionals, care providers, and stakeholders. American Journal of Public Health, 105, 881-890.

Yin, R. K. (2003) Case study research. Design and methods. Third Edit. Thousand Oakes, CA: SAGE Publications, Inc.

\section{Publication Acknowledgement}

This paper was originally published on Health Promotion International.

Sanchez-Pimienta, C. E., Masuda, J., \& M'Wikwedong Indigenous Friendship Centre. (2020). From controlling to connecting: M'Wikwedong as a place of urban 
Indigenous health promotion in Canada. Health Promotion International, 1-11. https://doi.org/10.1093/heapro/daaa066 